\title{
Efficacy and Tolerability of Oral Stevioside in Patients with Mild Essential Hypertension: A Two-Year, Randomized, Placebo-Controlled Study
}

\author{
Ming-Hsiung Hsieh, MD, ${ }^{1}$ Paul Chan, MD, PhD, ${ }^{1}$ Yuh-Mou Sue, MD, ${ }^{1}$ \\ Ju-Chi Liu, MD ${ }^{1}$ Toong Hua Liang, MD,${ }^{1}$ Tsuei-Yuen Huang, MD, ${ }^{2}$ \\ Brian Tomlinson, MD, ${ }^{3}$ Moses Sing Sum Chow, PharmD, ${ }^{3}$ \\ Pai-Feng Kao, MD, ${ }^{1}$ and Yi-Jen Chen, MD, $\mathrm{PhD}^{1}$ \\ ${ }^{1}$ Department of Medicine, Taipei Medical University-Wan Fang Hospital, Taipei, ${ }^{2}$ Division of \\ Cardiology, Chi-Mei Foundation Hospital, Tainan, Taiwan, and ${ }^{3}$ Division of Clinical \\ Pharmacology and School of Pharmacy, The Chinese University of Hong Kong, China
}

\begin{abstract}
Background: Stevioside, a natural glycoside isolated from the plant Stevia rebaudiana Bertoni, has been used as a commercial sweetening agent in Japan and Brazil for $>20$ years. Previous animal and human studies have indicated that stevioside has an antihypertensive effect.

Objectives: This study was undertaken to investigate the long-term (2-year) efficacy and tolerability of stevioside in patients with mild essential hypertension. Secondary objectives were to determine the effects of stevioside on left ventricular mass index (LVMI) and quality of life (QOL).

Methods: This was a multicenter, randomized, double-blind, placebocontrolled trial in Chinese men and women aged between 20 and 75 years with mild essential hypertension (systolic blood pressure [SBP] 140-159 mm Hg and diastolic blood pressure [DBP] 90-99 mm Hg). Patients took capsules containing $500 \mathrm{mg}$ stevioside powder or placebo 3 times daily for 2 years. Blood pressure was measured at monthly clinic visits; patients were also encouraged to monitor blood pressure at home using an automated device. LVMI was determined by 2-dimensional echocardiography at baseline and after 1 and 2 years of treatment. QOL was assessed using the Medical Outcomes Study 36-Item Short-Form Health Survey. Electrocardiographic, laboratory, and QOL parameters were assessed at the beginning of treatment, and at 6 months, 1 year, and 2 years.

Results: One hundred seventy-four patients ( 87 men, 87 women) were enrolled in the study, and 168 completed it: 82 (42 men, 40 women; mean [SD] age, 52 [7]
\end{abstract}

Accepted for publication September 11, 2003.

Printed in the USA. Reproduction in whole or part is not permitted.

$0|49-29| 8 / 03 / \$ 19.00$ 
years) in the stevioside group and 86 (44 women, 42 men; mean age, 53 [7] years) in the placebo group. After 2 years, the stevioside group had significant decreases in mean (SD) SBP and DBP compared with baseline (SBP, from 150 [7.3] to 140 [6.8] mm Hg; DBP, from 95 [4.2] to 89 [3.2] mm Hg; $P<0.05$ ) and compared with placebo $(P<0.05)$. Based on patients' records of self-monitored blood pressure, these effects were noted beginning $\sim 1$ week after the start of treatment and persisted throughout the study. There were no significant changes in body mass index or blood biochemistry, and the results of laboratory tests were similar in the 2 groups throughout the study. No significant difference in the incidence of adverse effects was noted between groups, and QOL scores were significantly improved overall with stevioside compared with placebo $(P<0.001)$. Neither group had a significant change in mean LVMI. However, after 2 years, 6 of 52 patients (11.5\%) in the stevioside group had left ventricular hypertrophy (LVH), compared with 17 of 50 patients $(34.0 \%)$ in the placebo group $(P<0.001)$. Of those who did not have LVH at baseline, 3 of 46 patients (6.5\%) in the stevioside group had developed LVH after 2 years, compared with 9 of 37 patients $(24.3 \%)$ in the placebo group $(P<0.001)$.

Conclusions: In this 2-year study in Chinese patients with mild hypertension, oral stevioside significantly decreased SBP and DBP compared with placebo. QOL was improved, and no significant adverse effects were noted. (Clin Ther. 2003;25: 2797-2808) Copyright (C) 2003 Excerpta Medica, Inc.

Key words: stevioside, hypertension, adverse effect, quality of life.

\section{INTRODUCTION}

Hypertension is one of the most important modifiable risk factors for coronary heart disease (the leading cause of death in the United States and many European nations), stroke (the third leading cause), congestive heart failure, end-stage renal disease, and peripheral vascular disease. ${ }^{1-4}$ Improvements in the identification and treatment of hypertension have contributed to a major reduction in the incidence of cardiovascular disease in many countries. ${ }^{5,6}$ Despite these advances in the detection and pharmacologic treatment of hypertension, inadequate blood pressure control continues to be a major public health problem. ${ }^{7}$ Compliance with antihypertensive therapy may be an important barrier to optimal blood pressure control, as some antihypertensive drug treatments can have a negative impact on quality of life (QOL). ${ }^{8,9}$ Development of new antihypertensive agents that have good efficacy and tolerability and that could also be regarded as natural products would be of considerable clinical interest, particularly in China, where herbal preparations are viewed favorably by patients.

Stevioside is a natural glycoside isolated from the plant Stevia rebaudiana Bertoni (Figure). It has been used as a commercial sweetening agent in Japan and Brazil 


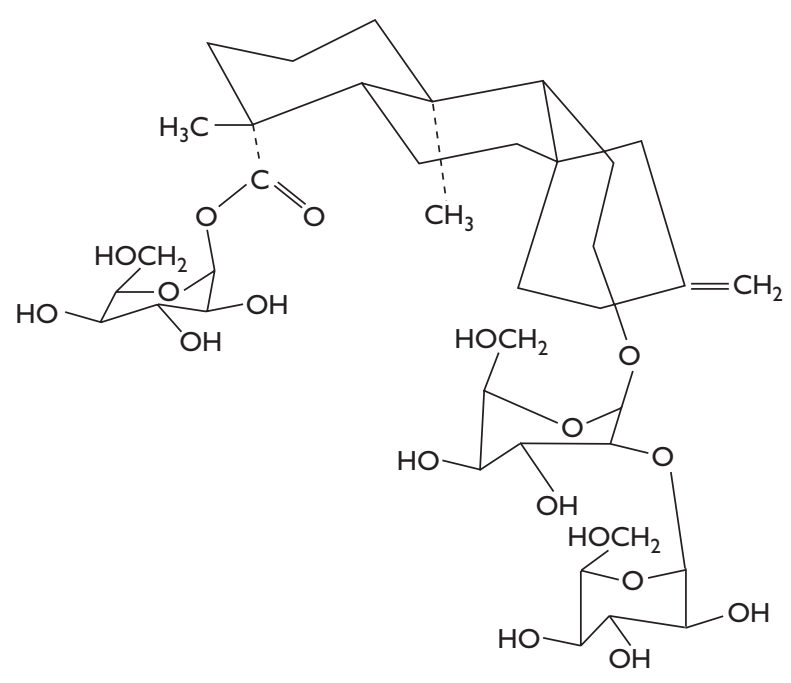

Figure. Chemical structure of stevioside.

for $>20$ years. ${ }^{10,11}$ Previous studies have shown that purified stevioside induces blood pressure reduction, diuresis, and natriuresis in rats. ${ }^{12}$ Intravenous administration of stevioside resulted in a clinically significant hypotensive effect in spontaneously hypertensive rats, without adverse effects on heart rate or serum catecholamine levels. ${ }^{13}$ In addition, previous animal studies found that stevioside had a short duration of action. ${ }^{14,15}$ In $>20$ years of use as a natural sweetener, stevioside has not been associated with significant adverse effects, which supports its tolerability during long-term use in humans. Recent preliminary data have shown its short-term effectiveness and tolerability in patients with hypertension. ${ }^{16}$

This study was undertaken to investigate the long-term efficacy and tolerability of stevioside in the treatment of patients with mild essential hypertension. Secondary objectives were to determine the effects of stevioside on left ventricular mass index (LVMI) and QOL.

\section{PATIENTS AND METHODS \\ Patient Population}

Eligible patients were Chinese men and women between the ages of 20 and 75 years with newly diagnosed mild (stage 1) essential hypertension, as defined in the sixth report of the Joint National Committee on Prevention, Detection, Evaluation, and Treatment of High Blood Pressure ${ }^{7}$ ( JNC VI)—sitting systolic blood pressure (SBP) between 140 and $159 \mathrm{~mm} \mathrm{Hg}$, and sitting diastolic blood pressure (DBP) between 90 and $99 \mathrm{~mm} \mathrm{Hg}$. They had to be otherwise healthy, with no target- 
organ damage caused by hypertension, no secondary causes of hypertension, and no cardiac disease, malignancies, significant renal impairment (serum creatinine concentration $>2.0 \mathrm{mg} / \mathrm{dL}$ ), or hepatic dysfunction.

\section{Study Design}

This was a 2-year, multicenter, double-blind, placebo-controlled trial. Following a 2-week placebo run-in phase during which no antihypertensive agents were given and blood pressure was monitored, a computer-generated randomization scheme was used to assign patients to receive stevioside capsules (Nan Kai Chemical Factory, Tien Jing, China) $500 \mathrm{mg}$ TID or matching placebo. Patients were asked to return for follow-up visits every month during active treatment for measurement of blood pressure and assessment of adverse effects.

Because these patients had stage 1 hypertension and were free of target-organ damage and other major cardiovascular risk factors, it was considered ethical for the study to include a placebo group. The JNC VI guidelines recommend drug treatment for patients with this degree of hypertension if blood pressure remains elevated after 12 months despite lifestyle modification, ${ }^{7}$ but other guidelines (eg, those of the World Health Organization-International Society for Hypertension ${ }^{17}$ ) suggest that such low-risk patients can be observed for a longer period. Furthermore, these patients were seen frequently, making it possible to initiate conventional antihypertensive medication if blood pressure was seen to be increasing.

The study protocol conformed to the ethical guidelines of the Declaration of Helsinki as amended in 1989 and was approved by the institutional review board of each participating center. All patients gave written informed consent.

\section{Study Assessments}

Blood pressure was measured in the clinic using a mercury sphygmomanometer. ${ }^{18}$ Trough values were the mean of 3 readings taken 10 minutes apart with the patient in the sitting position after a 15-minute rest. Patients were also encouraged to measure their blood pressure at home in the morning using an automated electronic device.

Patients were asked to abstain from heavy meals for 48 hours before a visit. Venous blood for laboratory tests was drawn between 8:00 and 10:00 Am after an overnight fast. Blood was collected into the appropriate tubes for determination of glucose, cholesterol, triglyceride, and electrolyte concentrations; renal function (uric acid, blood urea nitrogen, and creatinine); alanine aminotransferase (ALT); and aspartate aminotransferase (AST). Glucose, uric acid, blood urea nitrogen, creatinine, ALT, and AST were measured using a Monarch Autoanalyzer system (Instrumentation Laboratories, Lexington, Massachusetts). Total cholesterol and triglycerides were measured enzymatically using commercially available kits (Boehringer Mannheim GmbH, Mannheim, Germany). The high-density lipopro- 
tein cholesterol concentration was determined by precipitation, and the low-density lipoprotein cholesterol concentration was calculated using the Friedewald approximation.

LVMI was determined by 2-dimensional echocardiography (Sonos 5500 imaging system, Hewlett-Packard Company, Palo Alto, California) at baseline and after 1 and 2 years of treatment using the area-length algorithm. ${ }^{19}$ The individual who assessed the echocardiograms was blinded to treatment.

QOL was assessed using the Medical Outcomes Study 36-Item Short-Form Health Survey (SF-36), ${ }^{20}$ which consists of 8 subscales-physical functioning, role limitation-physical, bodily pain, general health perception, vitality, social functioning, role limitation-emotional, and mental health. Respondents are asked to evaluate their own health during the past 30 days. Each scale is scored from 0 (poorest health) to 100 (optimal health). The psychometric properties of the SF-36 have been examined extensively and found to be valid and reliable. ${ }^{21,22}$ The questionnaire has been shown to distinguish healthy from chronically ill individuals and to differentiate between patients with various types of chronic conditions. ${ }^{22}$

Electrocardiography, laboratory tests, and QOL assessments were performed at the beginning of treatment and at 6 months, 1 year, and 2 years. In addition, compliance was monitored by capsule counts at each visit.

\section{Statistical Analysis}

The primary efficacy end point was reduction in trough SBP and DBP at 2 years in the stevioside group compared with the placebo group using last-observationcarried-forward methodology. All statistical tests were 2-sided. Data are reported as mean [SD]. Significance was set at $P<0.05$ for differences between the activetreatment and placebo groups.

Based on $\alpha=0.05$ and an SD of $12 \mathrm{~mm} \mathrm{Hg}$, it was determined that 62 patients per group would provide $90 \%$ power to detect a difference in the primary efficacy end point of $7 \mathrm{~mm} \mathrm{Hg}$. Because of a scarcity of data specific to patients with stage 1 hypertension, this estimated SD was based on a previous study in patients with stage 1 hypertension. ${ }^{23}$

An analysis of variance (ANOVA) model in SAS (SAS Institute Inc., Cary, North Carolina) with site and treatment as factors was used to compare between-group changes in trough SBP and DBP; echocardiography and QOL measurements; and heart rate, body mass index (BMI), and electrocardiographic findings. The proportion of responders (those achieving an SBP $<140 \mathrm{~mm} \mathrm{Hg}$ or DBP $<90 \mathrm{~mm} \mathrm{Hg}$, or a $10 \%$ reduction in SBP or DBP from baseline) was calculated for each treatment group. ANOVA was used to assess the effects of BMI and blood pressure at baseline on the change from baseline in LVMI. Echocardiographic findings were analyzed only if a patient had both baseline and end-of-study examinations that were of acceptable technical quality. The Fisher exact test was used for comparisons of efficacy and safety between treatment groups. 


\section{RESULTS}

\section{Baseline Characteristics}

One hundred seventy-four patients ( 87 men, 87 women) were enrolled in the study, and 168 completed it: 82 (42 men, 40 women; mean [SD] age, 52 [7] years) in the stevioside group and 86 (44 women, 42 men; mean age, 53 [7] years) in the placebo group. Six patients ( 3 in each group) were withdrawn before the last scheduled study visit for loss to follow-up ( 1 in each group) and adverse effects ( 2 in each group). These patients were not included in the statistical analyses. Patients' baseline clinical and biochemical characteristics were similar at randomization (Table I).

Based on the analysis of data from patients with acceptable baseline and endof-study echocardiographic examinations (52 stevioside, 50 placebo), mean (SD)

Table I. Characteristics of the stevioside and placebo groups at baseline and end point. Values are mean (SD) unless otherwise indicated.

\begin{tabular}{|c|c|c|c|c|}
\hline & \multicolumn{2}{|c|}{ Stevioside } & \multicolumn{2}{|c|}{ Placebo } \\
\hline & Baseline & End Point & Baseline & End Point \\
\hline \multicolumn{5}{|l|}{ Sex, no. } \\
\hline Male & 43 & 42 & 44 & 42 \\
\hline Female & 42 & 40 & 45 & 44 \\
\hline Age, y & $5 \mid(6)$ & $52(7)$ & $52(8)$ & $53(7)$ \\
\hline $\mathrm{BMI}, \mathrm{kg} / \mathrm{m}^{2}$ & $22.9(2.6)$ & $23.0(2.0)$ & $23.8(2.6)$ & $23.6(2.4)$ \\
\hline SBP, mm Hg & $150(7.3)$ & $140(6.8)^{*+1}$ & $149(6.0)$ & $150(7.0)$ \\
\hline $\mathrm{DBP}, \mathrm{mm} \mathrm{Hg}$ & $95(4.2)$ & $89(3.2)^{*}+$ & $96(4.2)$ & $95(4.8)$ \\
\hline Heart rate, beats/min & $66.4(5.8)$ & $68.4(7.0)$ & $68.4(6.8)$ & $68.4(7.8)$ \\
\hline \multicolumn{5}{|l|}{ Serum values } \\
\hline Creatinine, $\mathrm{mg} / \mathrm{dL}$ & $1.2(0.4)$ & $1.2(0.5)$ & $1.2(0.3)$ & $1.3(0.4)$ \\
\hline $\mathrm{CPK}, \mathrm{U} / \mathrm{L}$ & $65(7)$ & $59(6)$ & $62(6)$ & $58(8)$ \\
\hline AST, U/L & $22(5)$ & $19(6)$ & $20(6)$ & $18(7)$ \\
\hline $\mathrm{ALT}, \mathrm{U} / \mathrm{L}$ & $20(4)$ & $18(6)$ & $18(5)$ & $20(6)$ \\
\hline Sodium, mEq/L & | $39.5(4.8)$ & | $40.2(4.3)$ & $138(4.8)$ & $141.8(3.6)$ \\
\hline Potassium, mEq/L & $4.5(0.4)$ & $4.3(0.5)$ & $4.3(0.4)$ & $4.5(0.3)$ \\
\hline Chloride, mEq/L & $99.8(7.8)$ & $98.3(6.8)$ & $100.4(10.8)$ & $100.8(12.0)$ \\
\hline \multicolumn{5}{|l|}{ Plasma values, mg/dL } \\
\hline Glucose & $100.8(11.2)$ & $101.4(10.8)$ & $102.5(12.6)$ & $108.2(11.8)$ \\
\hline Total cholesterol & $200.4(26.4)$ & $198.6(27.4)$ & $202.2(28.2)$ & $203.3(29.4)$ \\
\hline HDL-C & $49.8(19.2)$ & $50.5(\mid 8.2)$ & $49.9(\mid 8.6)$ & $50.2(20.5)$ \\
\hline Triglycerides & $130.6(30.2)$ & $128(36.6)$ & 133.5 (37.1) & $126.6(41.9)$ \\
\hline
\end{tabular}

$\mathrm{BMI}=$ body mass index; $\mathrm{SBP}=$ systolic blood pressure; $\mathrm{DBP}=$ diastolic blood pressure; $\mathrm{CPK}=$ creatinine phosphokinase; $\mathrm{AST}$ = aspartate aminotransferase; $\mathrm{ALT}=$ alanine aminotransferase; HDL-C = high-density lipoprotein cholesterol. ${ }^{*} P<0.05$ versus baseline.

$\dagger P<0.05$ versus placebo end point. 
LVMI was also similar at baseline in the 2 groups (stevioside, $92.4[16.8] \mathrm{g} / \mathrm{m}^{2}$; placebo, 93.4 [18.9] $\mathrm{g} / \mathrm{m}^{2}$ ). Left ventricular hypertrophy (LVH) has been defined as an LVMI $>116 \mathrm{~g} / \mathrm{m}^{2}$ for men and an LVMI $>104 \mathrm{~g} / \mathrm{m}^{2}$ for women. ${ }^{24}$ Based on these criteria, LVH was present at baseline in 8 patients in the stevioside group (15\%) and 12 patients (24\%) in the placebo group.

At baseline, the mean (SD) total score on the SF-36 was 68.8 (16.8) in the stevioside group and 67.4 (20.8) in the placebo group. There were no significant between-group differences in scores on any SF-36 subscale at baseline. In both groups, scores were highest on the physical functioning subscale and lowest on the general health perception subscale.

\section{Compliance}

All patients who completed the study followed the prescribed treatment schedule throughout the 2-year treatment period, and the degree of compliance was similar in the 2 groups. In the stevioside group, the mean (SD) intake of the planned number of capsules was 96\% (3\%) following randomization and 93\% (3\%) during treatment. In the placebo group, mean capsule intake was 95\% (4\%) following randomization and 92\% (3\%) during treatment.

\section{Efficacy}

Table I summarizes baseline and end-point blood pressure data for the 2 groups. After 2 years, mean [SD] SBP and DBP were significantly reduced from baseline in the stevioside group (SBP, from 150 [7.3] to 140 [6.8] mm Hg; DBP, from 95 [4.2] to 89 [3.2] mm Hg; $P<0.05$ ), representing respective reductions of 10 and $6 \mathrm{~mm} \mathrm{Hg}$. These reductions were also significant compared with the placebo group $(P<0.05)$. Based on patients' records of self-monitored blood pressure, the reductions began $\sim 1$ week after initiation of treatment and persisted throughout the treatment period (data not shown).

\section{Left Ventricular Mass Index}

After 2 years of treatment, neither group had a significant change from baseline in mean (SD) LVMI (stevioside, $-1.2[2.4] \mathrm{g} / \mathrm{m}^{2}$; placebo, $+2.0[4.8] \mathrm{g} / \mathrm{m}^{2}$ ). However, after 2 years, 6 of 52 patients (11.5\%) in the stevioside group had LVH, compared with 17 of 50 patients $(34.0 \%)$ in the placebo group $(P<0.001)$. Of those who did not have LVH at baseline, 3 of 46 patients (6.5\%) in the stevioside group had LVH after 2 years of treatment, compared with 9 of 37 patients $(24.3 \%)$ in the placebo group $(P<0.001)$.

\section{Quality of Life}

After 2 years of treatment, the stevioside group reported significantly higher total scores $(P<0.05)$ and scores on all SF-36 subscales except mental health (from 
$P<0.001$ to $P<0.05$ ) compared with placebo (Table II). The greatest improvements from baseline were seen in scores on the bodily pain and general health perception subscales. No significant changes in any subscale score were reported in the placebo group.

\section{Tolerability}

Stevioside was well tolerated. Eight patients in each group reported minor adverse effects, and only 2 in each group discontinued treatment. The types and incidence of adverse effects were similar between the active-treatment and placebo groups (Table III). Shortly after the initiation of treatment, 4 patients in the stevioside group experienced adverse effects (abdominal fullness, myalgia, nausea, and asthenia), but all symptoms disappeared after 1 week of treatment.

There were no significant changes in body weight or biochemical parameters, and the results of laboratory tests were similar in the 2 groups throughout the study. No cardiovascular events or mortality occurred in either group.

\section{DISCUSSION}

Achievement of target blood pressure requires high compliance with medication intake, and only $20 \%$ of all hypertensive patients achieve the required level of compliance. ${ }^{7}$ Because low compliance remains an important cause of poor blood pressure control, ${ }^{25}$ hypertension has been used as a model for understanding

Table II. Results of the Medical Outcomes Study 36-Item Short-Form Health Survey. Values are mean (SD).

\begin{tabular}{|c|c|c|c|c|}
\hline & \multicolumn{2}{|c|}{ Stevioside $(\mathrm{n}=82)$} & \multicolumn{2}{|c|}{ Placebo $(n=86)$} \\
\hline & Baseline & End Point & Baseline & End Point \\
\hline Total score & $68.8(16.8)$ & $78.4(15.3)^{\dagger}$ & $67.4(20.8)$ & $66.8(18.6)$ \\
\hline Physical functioning & $84.8(12.6)$ & $92.3(7.2)^{\ddagger}$ & $86.5(\mid 4.1)$ & $84.6(12.6)$ \\
\hline Role limitation-physical & $62.8(28.5)$ & $83.3(12.3)^{\S}$ & $64.2(34.7)$ & $63.8(30.0)$ \\
\hline Bodily pain & $65.6(22.8)$ & $76.6(17.6)^{\dagger}$ & $67.8(30.7)$ & $66.4(28.3)$ \\
\hline General health perception & $57.3(23.8)$ & $79.9(18.2)^{\S}$ & $58.4(27.7)$ & $56.5(25.8)$ \\
\hline Vitality & $60.5(25.4)$ & $72.2(10.5)^{\dagger}$ & $60.7(26.0)$ & $61.3(23.9)$ \\
\hline Social functioning & $66.2(18.6)$ & $88.3(15.4)^{\S}$ & $66.7(19.8)$ & $65.8(18.3)$ \\
\hline Role limitation-emotional & $58.1(27.8)$ & $80.6(11.5)^{\S}$ & $59.3(29.4)$ & $58.6(28.6)$ \\
\hline Mental health & $68.2(24.5)$ & $69.0(25.6)$ & $68.4(29.2)$ & $67.6(28.4)$ \\
\hline
\end{tabular}

*Each subscale is scored from 0 (poorest health) to 100 (optimal health).

${ }^{\dagger} p<0.01$ versus placebo.

$\ddagger P<0.05$ versus placebo.

$\S p<0.00$ I versus placebo. 
Table III. Adverse effects in the stevioside and placebo groups.

\begin{tabular}{lcc}
\hline Adverse Effect & $\begin{array}{c}\text { Stevioside } \\
(\mathrm{n}=85)\end{array}$ & $\begin{array}{c}\text { Placebo } \\
(\mathrm{n}=89)\end{array}$ \\
\hline Abdominal fullness & 2 & 2 \\
Nausea & $2^{*}$ & $2^{*}$ \\
Asthenia & 1 & $2^{*}$ \\
Dizziness & $1^{*}$ & 1 \\
Headache & 1 & 1 \\
Myalgia & 1 & 0 \\
\hline
\end{tabular}

*This event was of sufficient severity for treatment to be discontinued in I patient.

compliance. ${ }^{26}$ One of the adverse effects that is known to affect adherence to antihypertensive therapy is sexual dysfunction, ${ }^{27-30}$ and even the expectation that sexual function will be impaired may discourage a large number of patients from seeking appropriate antihypertensive therapy. ${ }^{31}$ In the present study, stevioside was effective in lowering blood pressure and had a positive effect on QOL, as indicated by SF-36 scores and the absence of complaints about sexual function.

Another important reason for poor compliance with antihypertensive regimens is the indefinite duration of therapy, ${ }^{31-33}$ and one of the goals of antihypertensive therapy is to minimize the adverse impact of this factor on QOL. ${ }^{34}$ The need for multiple medications is an additional complicating factor ${ }^{26}$; the compliance rate decreases as the number of daily medications increases. Previous investigators have found that compliance with antihypertensive medications improved from 59\% with a thrice-daily regimen to $86 \%$ with a once-daily regimen, and that there was no significant difference in compliance between once- and twice-daily regimens. ${ }^{35}$

Because stevioside has been found to have a short duration of action, ${ }^{13}$ stevioside capsules were administered 3 times daily in the present study. Despite the thrice-daily regimen, compliance was $>90 \%$. The use of alternative or complementary (traditional) medicine is very common in the Chinese population, and adherence to traditional medicines tends to be better. Because patients in this study were told that stevioside is a glycoside purified from a plant, they may have regarded it as a traditional medicine.

The active-treatment group had a significant reduction in blood pressure over the study period $(P<0.05)$. It is noteworthy that blood pressure began to decrease $\sim 1$ week after the initiation of stevioside therapy. Although the mechanism underlying stevioside's antihypertensive effect is not fully understood, the antihypertensive response to stevioside appears to occur through a mechanism of calcium-channel antagonism similar to that of verapamil. ${ }^{36-38}$ It has been re- 
ported that stevioside is capable of inhibiting calcium influx in rat smooth muscle cells. ${ }^{39}$

The tolerability of stevioside was similar to that of placebo in this study. Several studies have examined the effects of various antihypertensive drugs on QOL. ${ }^{40-44}$ In the present study, stevioside treatment was associated with significant improvement in QOL. The most marked improvements compared with placebo were seen in scores on 4 domains of the SF-36 (social functioning, general health perception, role limitation-emotional, and role limitation-physical).

\section{CONCLUSIONS}

In this 2-year study in Chinese patients with mild hypertension, oral stevioside significantly decreased SBP and DBP compared with placebo. QOL was improved, and no significant adverse effects were noted.

\section{REFERENCES}

1. Kannel WB, Belanger AJ. Epidemiology of heart failure. Am Heart J. 1991;121:951-957.

2. Klag MJ, Whelton PK, Randall BL, et al. Blood pressure and end-stage renal disease in men. N Engl J Med. 1996;334:13-18.

3. MacMahon S, Peto R, Cutler J, et al. Blood pressure, stroke, and coronary heart disease. Part 1. Prolonged differences in blood pressure: Prospective observational studies corrected for the regression dilution bias. Lancet. 1990;335;765-774.

4. Stamler J, Stamler R, Neaton JD. Blood pressure, systolic and diastolic, and cardiovascular risks. US population data. Arch Intern Med. 1993;153:598-615.

5. The 1988 report of the Joint National Committee on Detection, Evaluation, and Treatment of High Blood Pressure. Arch Intern Med. 1988;148:1023-1038.

6. Collins R, Peto R, MacMahon S, et al. Blood pressure, stroke, and coronary heart disease. Part 2. Short-term reductions in blood pressure: Overview of randomised drug trials in their epidemiological context. Lancet. 1990;335:827-838.

7. The sixth report of the Joint National Committee on Prevention, Detection, Evaluation, and Treatment of High Blood Pressure. Arch Intern Med. 1997;157:2413-2446.

8. Bulpitt CJ. Quality of life in antihypertensive patients. In: Amery A, ed. Hypertensive Cardiovascular Disease: Pathophysiology and Treatment. Boston: Kluwer Publishers; 1982:929-948.

9. Najman JM, Levine S. Evaluating the impact of medical care and technologies on the quality of life: A review and critique. Soc Sci Med. 1981;15:107-115.

10. Melis MS, Sainati AR. Participation of prostaglandins in the effect of stevioside on rat renal function and arterial pressure. Bras J Med Biol Res. 1991;24:1269-1276.

11. Melis MS, Macial RE, Sainati AR. Effects of indomethacin on the action of stevioside on mean arterial pressure and on renal function in rats. IRCS Med Sci. 1985;13:1230-1231.

12. Boeckh EMA, Humboldt G. Cardio-circulatory effects of total water extract in normal individuals and of stevioside in rats [in Portuguese]. Cienc Cultura. 1981;32:208-210. 
13. Chan P, Xu DY, Liu JC, et al. The effect of stevioside on blood pressure and plasma catecholamines in spontaneously hypertensive rats. Life Sci. 1998;63:1679-1684.

14. Kao PF, Hsu YH, Lee CN, et al. Hypotensive effect of intravenous stevioside in conscious and anesthetized animals. N Taipei J Med. 2001;3:176-181.

15. Hsu YH, Liu JC, Kao PF, et al. Antihypertensive effect of stevioside in different strains of hypertensive rats. Zhonghua Yi Xue Za Zhi (Taipei). 2002;65:1-6.

16. Chan P, Tomlinson B, Chen YJ, et al. A double-blind placebo-controlled study of the effectiveness and tolerability of oral stevioside in human hypertension. Br J Clin Pharmacol. 2000;50:215-220.

17. Guidelines Subcommittee. 1999 World Health Organization-International Society for Hypertension Guidelines for the Management of Hypertension. J Hypertens. 1999;17: 151-183.

18. Frohlich ED, Grim C, Labarthe DR, et al. Report of a special task force appointed by the steering committee, American Heart Association: Recommendations for human blood pressure determinations by sphygmomanometers. Hypertension. 1988;11:209A-222A.

19. Devereux RB, Reichek N. Echocardiographic determination of left ventricular mass in man. Anatomic validation of the method. Circulation. 1977;55:613-618.

20. Ware JE Jr, Sherbourne CD. The MOS 36-Item Short-Form Health Survey (SF-36). I. Conceptual framework and item selection. Med Care. 1992;30:473-483.

21. McHorney CA, Ware JE Jr, Raczek AE. The MOS 36-Item Short-Form Health Survey (SF-36): II. Psychometric and clinical tests of validity in measuring physical and mental health constructs. Med Care. 1993;31:247-263.

22. Dupuy HJ. The Psychological General Well-Being (PGWB) Index. In: Wenger NK, Mattson ME, Furberg CD, Elinson J, eds. Assessment of Quality of Life in Clinical Trials of Cardiovascular Therapies. New York: Le Jacq Publishing; 1984:170-183.

23. Black HR, Elliott WJ, Weber MA, et al. One-year study of felodipine or placebo for stage I isolated systolic hypertension. Hypertension. 2001;38:1118-1123.

24. Devereux RB, Dahlof B, Levy D, Pfeffer MA. Comparison of enalapril versus nifedipine to decrease left ventricular hypertrophy in systemic hypertension (the PRESERVE trial). Am J Cardiol. 1996;78:61-65.

25. Haynes RB. Determinants of compliance: The disease and the mechanics of treatment. In: Haynes PB, Taylor DW, Sackett DL, eds. Compliance in Health Care. Baltimore: Johns Hopkins University Press; 1979:49-62.

26. Sharkness CM, Snow DA. The patient's view of hypertension and compliance. Am J Prev Med. 1992;8:141-146.

27. Smith PJ, Talbert RL. Sexual dysfunction with antihypertensive and antipsychotic agents. Clin Pharm. 1986;5:373-384.

28. Stevenson JG, Umstead GS. Sexual dysfunction due to antihypertensive agents. Drug Intell Clin Pharmacol. 1984;18:113-121.

29. Trimmer E. Sex problems in practice. Iatrogenic sex problems. Br Med J (Clin Res Ed). 1981;283:953-955. 
30. Papadopoulos C. Cardiovascular drugs and sexuality: A cardiologist's review. Arch Intern Med. 1980;140:1341-1345.

31. Moss HB, Procci WR. Sexual dysfunction associated with oral antihypertensive medication: A critical survey of the literature. Gen Hosp Psychiatry. 1982;4:121-129.

32. Sackett DL, Haynes RB, Gibson ES, et al. Randomised clinical trial of strategies for improving medication compliance in primary hypertension. Lancet. 1975;1:1205-1207.

33. Hedstrand $\mathrm{H}$, Aberg $\mathrm{H}$. Treatment of hypertension in middle-aged men. A feasibility study in a community. Acta Med Scand. 1976;199:281-288.

34. Clark LT. Improving compliance and increasing control of hypertension: Needs of special hypertensive populations. Am Heart J. 1991;121:664-669.

35. Eisen SA, Miller DK, Woodward RS, et al. The effect of prescribed daily dose frequency on patient medication compliance. Arch Intern Med. 1990;150:1881-1884.

36. Melis MS, Sainati AR. Effect of calcium and verapamil on renal function of rats during treatment with stevioside. J Ethnopharmacol. 1991;33:257-262.

37. Evaluation of certain food additives. Fifty-first report of the Joint FAO/WHO Expert Committee on Food Additives. World Health Organ Tech Rep Ser. 2000;891:i-viii, 1-168.

38. Huxtable RJ. Pharmacology and toxicology of stevioside, rebaudioside A, and stevial. In: Kinghorn D, ed. The Genus Stevia. New York: Taylor and Francis; 2002:160-177.

39. Lee CN, Wong KL, Liu JC, et al. Inhibitory effect of stevioside on calcium influx to produce antihypertension. Planta Medica. 2001;67:796-799.

40. Beto JA, Bansal VK. Quality of life in treatment of hypertension: A metaanalysis of clinical trials. Am J Hypertens. 1992;5:125-133.

41. Hjemdahl P, Wiklund IK. Quality of life on antihypertensive drug therapy: Scientific end-point or marketing exercise? J Hypertens. 1992;10:1437-1446.

42. Jern S, Zanchetti A. The issue of quality of life in antihypertensive therapy. J Hum Hypertens. 1993; 7(Suppl 1):S46-S49.

43. Croog SH, Levine S, Testa MA, et al. The effects of antihypertensive therapy on the quality of life. N Engl J Med. 1986;341:1657-1664.

44. Testa MA, Anderson RB, Nackley JF, Hollenberg NK, for the Quality-of-Life Hypertension Group. Quality of life and antihypertensive therapy in men. A comparison of captopril with enalapril. N Engl J Med. 1993;328:907-913.

Address correspondence to: Paul Chan, MD, PhD, Department of Medicine, Taipei Medical University-Wan Fang Hospital, No 111, Hsing-Lung Road, Sec. 3, Wen-Shan District, Taipei City 117, Taiwan. E-mail: chanpaul@wanfang.gov.tw 